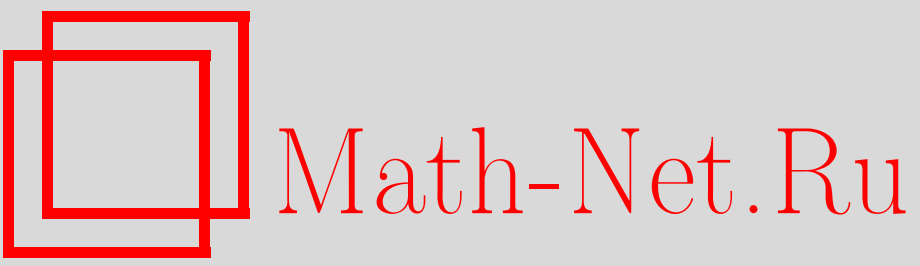

Т. В. Жуковская, И. Д. Серова, Об оценке решения краевой задачи для неявного дифференциального уравнения с отклоняющимся аргументом, Итоги науки и техн. Сер. Соврем. мат. и ее прил. Темат. обз., 2020, том 186, 38-44

DOI: https://doi.org/10.36535/0233-6723-2020-186-38-44

Использование Общероссийского математического портала Math-Net.Ru подразумевает, что вы прочитали и согласны с пользовательским соглашением

http://www.mathnet.ru/rus/agreement

Параметры загрузки:

IP : 54.174 .149 .18

26 апреля 2023 г., 12:44:40 


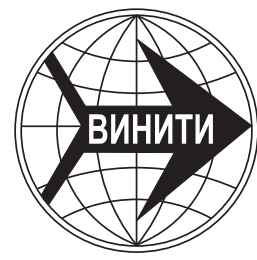

ИТОГИ НАУКИ И ТЕХНИКИ.

Современная математика и ее приложения.

Тематические обзоры.

Том 186 (2020). С. 38-44

DOI: $10.36535 / 0233-6723-2020-186-38-44$

УДК 517.91

\title{
ОБ ОЦЕНКЕ РЕШЕНИЯ КРАЕВОЙ ЗАДАЧИ \\ ДЛЯ НЕЯВНОГО ДИФФЕРЕНЦИАЛЬНОГО УРАВНЕНИЯ \\ С ОТКЛОНЯЮЩИМСЯ АРГУМЕНТОМ
}

\author{
(c) 2020 г. $\quad$ Т. В. ЖУКОВСКАЯ, И. Д. СЕРОВА
}

\begin{abstract}
АннотАция. Исследуется двухточечная краевая задача для неявного дифференциального уравнения с отклоняющимся аргументом. Получена теорема о существовании и оценке решения, аналогичная теореме Чаплыгина о дифференциальном неравенстве. Используются результаты об уравнениях с накрывающими и монотонными отображениями в частично упорядоченных пространствах, а также условия упорядоченного накрывания оператора Немыцкого в пространстве измеримых существенно ограниченных функций.
\end{abstract}

Ключевые слова: неявное дифференциальное уравнение, краевая задача, условие разрешимости, оценка решения, упорядоченно накрывающее отображение, дифференциальное неравенство.

\section{ON ESTIMATES OF SOLUTIONS OF BOUNDARY-VALUE PROBLEMS FOR IMPLICIT DIFFERENTIAL EQUATIONS WITH DEVIATING ARGUMENT}

\author{
(c) 2020 T. V. ZHUKOVSKAYA, I. D. SEROVA
}

\begin{abstract}
A two-point boundary-value problem for an implicit differential equation with a deviating argument is examined. An existence theorem and an estimate for the solution are obtained, which is similar to the Chaplygin theorem on differential inequalities. We use results on equations with covering and monotonic mappings in partially ordered spaces and conditions for ordered covering of the Nemytskii operator in the space of measurable essentially bounded functions.
\end{abstract}

Keywords and phrases: implicit differential equation, boundary-value problem, solvability condition, estimate of solution, ordered covering mapping, differential inequality.

AMS Subject Classification: 34A09, 34B15, 34A40, 47H07

1. Введение. В связи с развитием сложных робототехнических систем, исследованиями задач физики, механики и небесной механики усилился интерес к динамическим системам, описываемым неявными дифференциальными уравнениями. Анализ, геометрия, другие разделы современной математики предоставляют эффективные методы исследования неявных дифференциальных уравнений. Тем не менее, для неявных уравнений недостаточно подробно изучены краевые задачи и задачи управления, дифференциальные неравенства, условия устойчивости и некоторые другие проблемы. Новые возможности в исследовании этих задач открывают интенсивно развивающаяся

Работа выполнена при поддержке Российского фонда фундаментальных исследований (проект № 17-41$680975 p_{-}$a). 
теория накрывающих отображениях метрических и частично упорядоченных пространств. Методы исследования задачи Коши, краевых задач и задач управления, основанные на результатах о накрывающих отображениях метрических пространств разработаны в работах $[1,3,9]$

В работах $[4,5,16,17]$ определены и исследованы накрывающие отображения частично упорядоченных пространств. Для неявного дифференциального уравнения методами, основанными на упорядоченно накрывающих отображениях, в [7] получена теорема об оценке решения задачи Коши, в [13] - теорема об оценке решения краевой задачи. В [8] доказана теорема о неявном интегральном неравенстве. В работах $[11,12,14]$ упорядоченно накрывающие отображения применены к исследованию задачи Коши для неявного дифференциального уравнения с запаздывающим аргументом. В настоящей работе методами, основанными на упорядоченно накрывающих отображениях, исследуется краевая задача для неявного дифференциального уравнения с отклоняющимся аргументом.

2. Основные понятия. Здесь мы сформулируем утверждения об обобщенной обратной матрице и об отображениях частично упорядоченных пространств, на основании которых далее будут получены условия разрешимости краевых задач и даны оценки их решений.

2.1. Обобщенная обратная матрища. Напомним вначале некоторые известные сведения из линейной алгебры, необходимые в нашем исследовании (подробнее см. [10, § 51]).

Пусть задана вещественная матрица $A$. Будем писать $A \geqslant 0(A \leqslant 0)$, если все элементы матрицы $A$ неотрицательны (неположительны).

Обобщенно обратной или псевдообратной к матрице $A$ называется матрица $A^{+}$такая, что $A A^{+} A=A, A^{+} A A^{+}=A^{+}$и матрицы $A A^{+}$и $A^{+} A$ симметричны. Согласно теореме МураПенроуза [10, теорема 51.1.2] для любой матрицы $A$ существует единственная псевдообратная матрица $A^{+}$. При этом, если строки матрицы $A$ линейно независимы, то матрица $A A^{T}$ обратима и псевдообратная матрица задается формулой $A^{+}=A^{T}\left(A A^{T}\right)^{-1}$, где $A^{T}$ - транспонированная матрица.

Псевдообратные матрицы используются при решении систем уравнений. Рассмотрим матричное уравнение $A U=C$ относительно неизвестной матрицы $U$. Это уравнение разрешимо тогда и только тогда, когда (см. [10, теорема 51.2.2])

$$
A A^{+} C=C \text {. }
$$

При выполнении условия (1) общее решение рассматриваемого уравнения имеет вид

$$
U=A^{+} C+\left(E-A^{+} A\right) D,
$$

где $E$ - единичная матрица, а $D$ - произвольная матрица. Отметим, что в случае, когда строки матрицы $A$ линейно независимы, условие (1), очевидно, выполнено, то есть рассматриваемое уравнение разрешимо для любой правой части $C$, его решение определяется соотношением

$$
U=A^{T}\left(A A^{T}\right)^{-1} C+\left(E-A^{T}\left(A A^{T}\right)^{-1} A\right) D .
$$

2.2. Уравнения в частично упорядоченных пространствах. Теперь приведем сведения об отображениях, действующих в частично упорядоченных пространствах, существенно используемые в работе.

Пусть заданы частично упорядоченные пространства $X=(X, \preceq), Y=(Y, \preceq)$. Для элемента $u \in X$ определим множество $\mathcal{O}_{X}(u) \doteq\{x \in X: x \preceq u\}$.

Определение 1. Отображение $G: X \rightarrow Y$ называется антитонным на множестве $X_{0} \subset X$, если справедливо соотношение

$$
\forall x, u \in X_{0} x \preceq u \Rightarrow G(x) \succeq G(u) .
$$

Определение 2 (см. [7]). Отображение $G: X \rightarrow Y$ называется упорядоченно накрывающим множество $Y_{0} \subset Y$, если справедливо соотношение

$$
\forall u \in X \forall y \in Y_{0} y \preceq G(u) \Rightarrow \exists x \in X G(x)=y \& x \preceq u .
$$


Пусть заданы: элемент $y \in Y$ и отображение $\Psi: X^{2} \rightarrow Y$, являющееся по одному аргументу упорядоченно накрывающим некоторое множество из $Y$, а по другому - антитонным на некотором множестве из $X$. Определим отображение $F: X \rightarrow Y$ равенством

$$
F(x)=\Psi(x, x) \quad \forall x \in X
$$

и рассмотрим уравнение

$$
F(x)=y
$$

Сформулируем условия разрешимости этого уравнения.

Для отображение $\Psi$, элемента $y \in Y$ и произвольного множества $X_{0} \subset X$ определим совокупность $Z\left(\Psi, X_{0}, y\right)$ всех цепей $S \subset X_{0}$, удовлетворяющих условиям

$$
\forall x \in S \Psi(x, x) \succeq y, \forall x_{1}, x_{2} \in S x_{1} \prec x_{2} \Rightarrow \Psi\left(x_{1}, x_{2}\right) \preceq y .
$$

Теорема 1 (см. [12]). Пусть существует такой элемент $u_{0} \in X$, что справедливо неравенство

$$
\Psi\left(u_{0}, u_{0}\right) \succeq y,
$$

и выполнены следующие условия:

(а) при любом $x \in \mathcal{O}_{X}\left(u_{0}\right)$ отображсение $\Psi(\cdot, x): X \rightarrow Y$ упорядоченно накрывает множество $Y_{0} \doteq\{y\}$

(b) отображение $\Psi(x, \cdot): X \rightarrow Y$ при любом $x \in \mathcal{O}_{X}\left(u_{0}\right)$ является антитонным на множестве $\mathcal{O}_{X}\left(u_{0}\right)$

(с) любал иепь $S \in Z\left(\Psi, \mathcal{O}_{X}\left(u_{0}\right), y\right)$ ограничена снизу и имеет нижнюю границу $\omega \in X$, удовлетворяющую неравенству $\Psi(\omega, \omega) \succeq y$.

Тогда множество решений уравнения (2) не пусто, в нем существует минимальный элемент, который принадлежит множеству $\mathcal{O}_{X}\left(u_{0}\right)$.

Приведенное утверждение доказано в работе [12], оно аналогично более раннему результату, полученному в [7].

2.3. Условия упорядоченного накрывания оператора Немыикого. Для применения теоремы 1 к дифференциальным уравнениям требуются условия упорядоченного накрывания оператора суперпозиции (оператора Немыцкого). Сформулируем эти условия.

Обозначим $L_{\infty}^{n} \doteq L_{\infty}\left([a, b], \mathbb{R}^{n}\right)$ - пространство измеримых существенно ограниченных функций $x:[a, b] \rightarrow \mathbb{R}^{n}$ с нормой

$$
\|x\|_{L_{\infty}^{n}}=\text { vrai } \sup _{t \in[a, b]}|x(t)|_{\mathbb{R}^{n}} \quad \forall x \in L_{\infty} ;
$$

$L_{\infty}^{n}(\mathcal{B}) \doteq L_{\infty}([a, b], \mathcal{B})$ - подмножество пространства $L_{\infty}^{n}$, состоящее из функций со значениями в непустом множестве $\mathcal{B} \subset \mathbb{R}^{n} ; W^{m} \doteq W\left([a, b], \mathbb{R}^{m}\right)$ - множество измеримых функций $x:[a, b] \rightarrow$ $\mathbb{R}^{m}$. В множествах $L_{\infty}^{n}$ и $W^{m}$ считаем, что задан «естественный» порядок, то есть для функций $x, u$ полагаем $x \leqslant u$, если $x(t) \leqslant u(t)$ при п. в. (почти всех) $t \in[a, b]$.

Пусть задана удовлетворяющая условиям Каратеодори (измеримая по первому аргументу и непрерывная по второму аргументу) функция $g:[a, b] \times \mathbb{R}^{n} \rightarrow \mathbb{R}^{m}$. Определим соответствующий ей оператор Немыцкого, действующий в пространствах измеримых функций, равенством

$$
\left(N_{g} x\right)(t)=g(t, x(t)), t \in[a, b] .
$$

Зададим число $r \geqslant 0$ и обозначим через $g_{r}$ сужение функции $g$ на множество $[a, b] \times \mathcal{B}_{r}$, где $\mathcal{B}_{r} \doteq \mathcal{B}_{\mathbb{R}^{n}}(0, r)$ - это шар в $\mathbb{R}^{n}$ с центром в 0 радиуса $r$. Определим оператор Немыцкого, соответствующий функции $g_{r}$ :

$$
N_{g_{r}}: L_{\infty}^{n}\left(\mathcal{B}_{r}\right) \rightarrow W^{m}, \quad\left(N_{g_{r}} x\right)(t)=g_{r}(t, x(t)), \quad t \in[a, b] .
$$

Следующее утверждение позволяет установить связь между свойствами упорядоченного накрывания функции $g_{r}$ по второму аргументу и оператора Немыцкого $N_{g_{r}}$.

Теорема 2 (см. [7]). Пусть $y \in W^{m}$. Если при п. в. $t \in[a, b]$ функиия $g_{r}(t, \cdot): \mathcal{B}_{r} \rightarrow \mathbb{R}^{m}$ упорлдоченно накрывает одноточечное множество $Y_{0}(t) \doteq\{y(t)\} \subset \mathbb{R}^{m}$, то оператор Немыцкого $N_{g_{r}}: L_{\infty}^{n}\left(\mathcal{B}_{r}\right) \rightarrow W^{m}$ упорядоченно накрывает множество $Y_{0} \doteq\{y(\cdot)\} \subset W^{m}$. 
3. Краевая задача для неявного дифференциального уравнения. Теперь на основании теорем 1 и 2 сформулируем и докажем основной результат работы - утверждение о существовании и оценке решения краевой задачи для неявного дифференциального уравнения с отклоняющимся аргументом.

Через $A C_{\infty}^{n} \doteq A C_{\infty}\left([a, b], \mathbb{R}^{n}\right)$ будем обозначать пространство абсолютно непрерывных функций $x:[a, b] \rightarrow \mathbb{R}^{n}$, имеющих производную $\dot{x} \in L_{\infty}^{n} ;$ через $A C_{\infty}^{n}(\mathcal{B}) \doteq A C_{\infty}([a, b], \mathcal{B})$ - его подмножество, содержащее функции $x:[a, b] \rightarrow \mathbb{R}^{n}$ такие, что $\dot{x} \in L^{n}(\mathcal{B})$, где $\mathcal{B} \subset \mathbb{R}^{n}, \mathcal{B} \neq \emptyset$.

Пусть заданы матрицы $A, B \in \mathbb{R}^{k \times n}, k \leqslant n$, и вектор $C \in \mathbb{R}^{k}$. Будем предполагать, что ранг матрицы $A+B$ максимален, то есть $\operatorname{rank}(A+B)=k$. Пусть определены функции $h:[a, b] \rightarrow \mathbb{R}$, $f:[a, b] \times \mathbb{R}^{n} \times \mathbb{R}^{n} \rightarrow \mathbb{R}^{m}$. Рассмотрим краевую задачу для уравнения

$$
f(t, x(h(t)), \dot{x}(t))=0, t \in[a, b], x(s)=0, \text { если } s \notin[a, b],
$$

с условием

$$
A x(a)+B x(b)=C .
$$

Для произвольной функции $x:[a, b] \rightarrow \mathbb{R}^{n}$ положим

$$
\left(S_{h} x\right)(t)= \begin{cases}x(h(t)), & \text { если } h(t) \in[a, b], \\ 0, & \text { если } h(t) \notin[a, b] .\end{cases}
$$

Следуя формализации уравнений с отклоняющимся аргументом, предложенной Н. В. Азбелевым (см. [2]), будем записывать уравнение (4) в виде

$$
f\left(t,\left(S_{h} x\right)(t), \dot{x}(t)\right)=0, t \in[a, b],
$$

а под решением уравнения (4) будем понимать функцию $x \in A C_{\infty}^{n}$, удовлетворяющую (6) при п. в. $t \in[a, b]$.

Теорема 3. Пусть заданы функция $v_{0} \in A C_{\infty}^{n}$ и число $r \geqslant\left\|\dot{v}_{0}\right\|_{L_{\infty}^{n}}$. Пусть справедливы неравенства

$$
f\left(t,\left(S_{h} v_{0}\right)(t), \dot{v}_{0}(t)\right) \geqslant 0 \text { nрu n. в. } t \in[a, b], A v_{0}(a)+B v_{0}(a) \geqslant C,
$$

и выполнены условия:

(d) функиия $f(\cdot, x, u):[a, b] \rightarrow \mathbb{R}^{m}$ измерима при любых $x, u \in \mathbb{R}^{n}$;

(е) при п. в. $t \in[a, b]$ и любых $u \in \mathbb{R}^{n}$ функиия $f(t, \cdot, u): \mathbb{R}^{n} \rightarrow \mathbb{R}^{m}$ по каждому аргументу $x_{1}, \ldots, x_{n}$ не возрастает и непрерывна справа;

(f) имеют место соотношения

$$
\operatorname{rank}(A+B)=k,(A+B)^{+} \geqslant 0,(A+B)^{+} B \leqslant 0,
$$

где

$$
(A+B)^{+}=(A+B)^{T}\left((A+B)(A+B)^{T}\right)^{-1} ;
$$

(g) при п. в. $t \in[a, b]$ и любых $x \in \mathbb{R}^{n}$ функция $f(t, x, \cdot): \mathbb{R}^{n} \rightarrow \mathbb{R}^{m}$ непрерывна и ее сужение $f_{r}(t, x, \cdot): \mathcal{B}_{r} \rightarrow \mathbb{R}^{m}$ на шар $\mathcal{B}_{r} \doteq \mathcal{B}_{\mathbb{R}^{n}}(0, r)$ упорлдоченно накрывает множество $\{0\} \subset \mathbb{R}^{m} ;$

(h) функиия $h:[a, b] \rightarrow \mathbb{R}$ измерима.

Тогда существует решение $x \in A C_{\infty}^{n}\left(\mathcal{B}_{r}\right)$ задачи (4), (5), удовлетворяющее при n. в. $t \in[a, b]$ неравенствам $\dot{x}(t) \leqslant \dot{v}_{0}(t)$.

Доказательство. В силу предположения (е) при любом $u \in \mathbb{R}^{n}$ суперпозиция $f(\cdot, z(\cdot), u):[a, b] \rightarrow$ $\mathbb{R}^{m}$ измерима для любой измеримой функции $z:[a, b] \rightarrow \mathbb{R}^{n}$ (см., например, [15], а также $[8$, c. 110,111$])$. Поэтому согласно условию $(\mathrm{h})$ функция $f\left(\cdot,\left(S_{h} x\right)(\cdot), u\right):[a, b] \rightarrow \mathbb{R}^{m}$ измерима для любой непрерывной функции $x:[a, b] \rightarrow \mathbb{R}^{n}$. Теперь заметим, что функция $(t, u) \in$ $[a, b] \times \mathbb{R}^{n} \mapsto f\left(t,\left(S_{h} x\right)(t), u\right) \in \mathbb{R}^{m}$ удовлетворяет условиям Каратеодори, следовательно, для произвольных измеримой функции $u:[a, b] \rightarrow \mathbb{R}^{n}$ и непрерывной функции $x:[a, b] \rightarrow \mathbb{R}^{n}$ функция $f\left(\cdot,\left(S_{h} x\right)(\cdot), u(\cdot)\right):[a, b] \rightarrow \mathbb{R}^{m}$ также измерима. Отсюда следует, что при любом $x \in A C_{\infty}^{n}$ функция $f\left(\cdot,\left(S_{h} x\right)(\cdot), \dot{x}(\cdot)\right):[a, b] \rightarrow \mathbb{R}^{m}$ измерима. 
Преобразуем краевое условие (5) следующим образом:

$$
(A+B) x(a)=C-B(x(b)-x(a)) \Longleftrightarrow(A+B) x(a)=A+B \int_{a}^{b} \dot{x}(s) d s .
$$

Так как $\operatorname{rank}(A+B)=k$, это уравнение можно разрешить относительно $x(a)$ :

$$
x(a)=(A+B)^{+}\left(C-B \int_{a}^{b} \dot{x}(s) d s\right)+D-(A+B)^{+}(A+B) D,
$$

где $D \in \mathbb{R}^{n}$ - произвольный вектор, а матрица $(A+B)^{+}$определяется соотношением (8). Положим $D=0$ и рассмотрим краевую задачу для уравнения (4) с условием

$$
x(a)=(A+B)^{+}\left(C-B \int_{a}^{b} \dot{x}(s) d s\right) .
$$

Любое решение задачи (4), (9) является решением исходной задачи (4), (5). Докажем, что задача (4), (9) разрешима.

Обозначим через $f_{r}:[a, b] \times \mathbb{R}^{n} \times \mathcal{B}_{r} \rightarrow \mathbb{R}^{m}$ сужение исходной функции $f$ и определим для $f_{r}$ оператор Немыцкого

$$
N_{f_{r}}: W^{n} \times L_{\infty}^{n}\left(\mathcal{B}_{r}\right) \rightarrow W^{m}, \quad\left(N_{f_{r}}(y, x)\right)(t)=f_{r}(t, x(t), y(t)) .
$$

Тогда задачу (4), (9) запишем в виде уравнения

$$
N_{f_{r}}\left(y, S_{h}\left((A+B)^{+}\left(C-B \int_{a}^{b} y(s) d s\right)+\int_{a}^{(\cdot)} y(s) d s\right)\right)=0
$$

относительно неизвестной функции $y=\dot{x}, y \in L_{\infty}^{n}\left(\mathcal{B}_{r}\right)$. Докажем, что определенное равенством

$$
\Psi(y, u)=N_{f_{r}}\left(u, S_{h}\left((A+B)^{+}\left(C-B \int_{a}^{b} y(s) d s\right)+\int_{a}^{(\cdot)} y(s) d s\right)\right)
$$

отображение $\Psi: L_{\infty}^{n}\left(\mathcal{B}_{r}\right) \times L_{\infty}^{n}\left(\mathcal{B}_{r}\right) \rightarrow W^{m}$ удовлетворяет условиям теоремы 1 .

Положим $\Delta C \doteq A v_{0}(a)+B v_{0}(a)-C$. Согласно второму неравенству (7) имеем $\Delta C \geqslant 0$. Для производной $\dot{v}_{0}$ из неравенств $(7)$ и условия (е) следует

$$
\begin{aligned}
0 \leqslant N_{f_{r}}\left(\dot{v}_{0}, S_{h}\left((A+B)^{+}\left(C+\Delta C-B \int_{a}^{b} \dot{v}_{0}(s) d s\right)+\int_{a}^{(\cdot)} \dot{v}_{0}(s) d s\right)\right) \leqslant \\
\leqslant N_{f_{r}}\left(\dot{v}_{0}, S_{h}\left((A+B)^{+}\left(C-B \int_{a}^{b} \dot{v}_{0}(s) d s\right)+\int_{a}^{(\cdot)} \dot{v}_{0}(s) d s\right)\right)=\Psi\left(\dot{v}_{0}, \dot{v}_{0}\right) .
\end{aligned}
$$

Таким образом, выполнено неравенство (3).

Условие (a) теоремы 1 также выполнено, так как из условия (g) на основании теоремы 2 следует, что отображение $\Psi(\cdot, u)$ упорядоченно накрывает множество $\{0\} \in W^{m}$ при любом $u \in L_{\infty}^{n}\left(\mathcal{B}_{r}\right)$.

Согласно условиям (е) и (f) при п. в. $t \in[a, b]$ функция $f_{r}(t, \cdot, u(t)): \mathcal{B}_{r} \rightarrow \mathbb{R}^{m}$ не возрастает по каждому аргументу $x_{1}, \ldots, x_{n}$ для любого $u \in L_{\infty}^{n}\left(\mathcal{B}_{r}\right)$. Следовательно, отображение $\Psi(u, \cdot): L_{\infty}^{n} \rightarrow W^{m}$ антитонное, то есть условие (b) теоремы 1 выполнено. 
Проверим условие (с) теоремы 1 . Для этого определим произвольную цепь $S \subset L_{\infty}^{n}\left(\mathcal{B}_{r}\right)$ такую, чтобы для любого элемента $w \in S$ выполнялось неравенство $\Psi(w, w) \geqslant 0$, то есть

$$
N_{f_{r}}\left(w, S_{h}\left((A+B)^{+}\left(C-B \int_{a}^{b} w(s) d s\right)+\int_{a}^{(\cdot)} w(s) d s\right)\right) \geqslant 0 .
$$

Так как цепь $S$ ограничена снизу, то она имеет в $L_{\infty}^{n}\left(\mathcal{B}_{r}\right)$ точную нижнюю границу $\underline{w}$. Выделим из этой цепи невозрастающую последовательность $\left\{w_{i}\right\} \subset S$, такую, что $\inf \left\{w_{i}\right\}=\inf S=\underline{w}$ (см. [6, гл. IV, $\S 12$, следствие 7, с. 365$])$. Следовательно, при п. в. $t \in[a, b]$ имеют место равенства $\underline{w}(t)=\inf \left\{w_{i}(t)\right\}=\lim _{i \rightarrow \infty} w_{i}(t)$. Так как отображение $\Psi\left(w_{i}, \cdot\right): L_{\infty}^{n}\left(\mathcal{B}_{r}\right) \rightarrow W^{m}$ антитонное, имеем

$$
\begin{aligned}
N_{f_{r}}\left(w_{i}, S_{h}\left((A+B)^{+}\left(C-B \int_{a}^{b} \underline{w}(s) d s\right)+\int_{a}^{(\cdot)} \underline{w}(s) d s\right)\right) \geqslant \\
\geqslant N_{f_{r}}\left(w_{i}, S_{h}\left((A+B)^{+}\left(C-B \int_{a}^{b} w_{i}(s) d s\right)+\int_{a}^{(\cdot)} w_{i}(s) d s\right)\right) \geqslant 0 .
\end{aligned}
$$

Отсюда вследствие непрерывности функции $f$ по третьему аргументу (см. предположения $(\mathrm{g}))$ при п. в. $t \in[a, b]$ выполнено соотношение

$$
(\Psi(\underline{w}, \underline{w}))(t)=\lim _{i \rightarrow \infty} f\left(t,\left(S_{h}\left((A+B)^{+}\left(C-B \int_{a}^{b} \underline{w}(s) d s\right)+\int_{a}^{(\cdot)} \underline{w}(s) d s\right)\right)(t), w_{i}(t)\right) \geqslant 0 .
$$

Следовательно, условие (с) теоремы 1 выполнено.

Таким образом, установлена разрешимость задачи (4), (9), следовательно, и задачи (4), (5).

Замечание. Утверждение теоремы 3 можно уточнить, если одно из неравенств (7) вырождается в равенство. Если для $v_{0} \in A C_{\infty}^{n}$ при п. в. $t \in[a, b]$ выполнено $f\left(t,\left(S_{h} v_{0}\right)(t), \dot{v}_{0}(t)\right)=0$, то в условии (f) следует оставить только первое неравенство, а второе можно опустить. Если же выполнено $A v_{0}(a)+B v_{0}(a)=C$, то в условии (f) следует оставить только второе неравенство, а первое можно опустить. Утверждение теоремы 3 при этом останется справедливым.

В заключение рассмотрим краевую задачу с условием (5) для обыкновенного дифференциального уравнения

$$
f(t, x(t), \dot{x}(t))=0, t \in[a, b] .
$$

Достаточные условия ее разрешимости могут быть выведены из теоремы 3 , если принять $h(t)=t$.

Следствие. Пусть заданы функиия $v_{0} \in A C_{\infty}^{n}$ и число $r \geqslant\left\|\dot{v}_{0}\right\|_{L_{\infty}^{n}}$. Пусть справедливы неравенства

$$
f\left(t, v_{0}(t), \dot{v}_{0}(t)\right) \geqslant 0 \text { nри n. в. } t \in[a, b], A v_{0}(a)+B v_{0}(a) \geqslant C,
$$

и выполнены условия (d)-(g) теоремы 3.

Тогда существует решение $x \in A C_{\infty}^{n}\left(\mathcal{B}_{r}\right)$ задачи (10), (5), удовлетворяющее при n. в. $t \in[a, b]$ неравенству $\dot{x}(t) \leqslant \dot{u}_{0}(t)$.

Это утверждение было ранее получено в [13].

\section{СПИСОК ЛИТЕРАТУРЫ}

1. Аваков E. Р., Арутюнов А. В., Жуковский Е. С. Накрывающие отображения и их приложения к дифференциальным уравнениям, не разрешенным относительно производной// Диффер. уравн. 2009. - 45, № 5. - C. 613-634.

2. Азбелев Н. В., Максимов В. П., Рахматуллина Л. Ф. Введение в теорию функциональнодифференциальных уравнений. - М.: Наука, 1991. 
3. Арутюнов А. В., Жуковский Е. С., Жуковский C. Е. О корректности дифференциальных уравнений, не разрешенных относительно производной // Диффер. уравн. - 2011. - 47, № 11. - С. $1523-1537$.

4. Арутюнов A. В., Жуковский E. С., Жуковский C. Е. О точках совпадения отображений в частично упорядоченных пространствах// Докл. РАН. - 2013. - 453, № 5. - С. 475-478.

5. Арутюнов A. В., Жуковский Е. С., Жуковский C. Е. Точки совпадения многозначных отображений в частично упорядоченных пространствах// Докл. РАН. - 2013. - 453, № 6. - С. 595-598.

6. Данфорд Н., Швари, Дж. Линейные операторы. Т. 1. Общая теория. - М.: ИЛ, 1962.

7. Жуковский E. C. Об упорядоченно накрывающих отображениях и неявных дифференциальных неравенствах// Диффер. уравн. - 2016. - 52, № 12. - С. 1605-1621.

8. Жуковский E. C. Об упорядоченно накрывающих отображениях и интегральных неравенствах типа Чаплыгина// Алгебра анал. - 2018. - 30, № 1. - С. 96-127.

9. Жуковский E. C., Плужникова E. A. Накрывающие отображения в произведении метрических пространств и краевые задачи для дифференциальных уравнений, не разрешенных относительно производной// Диффер. уравн. - 2013. - 49, № 4. - С. 439-455.

10. Прасолов В. В. Задачи и теоремы линейной алгебры. - М.: МЦНМО, 2015.

11. Серова И. Д. Об оценках решения неявного функционально-дифференциального уравнения// Прикл. мат. вопр. управл. - 2017. - 2. - С. 85-93.

12. Серова И. Д. О неявных дифференциальных неравенствах с отклоняющимся аргументом// Вестн. Тамбов. ун-та. Сер. Естеств. техн. науки. - 2017. - 21, № 3. - С. 571-578.

13. Серова И. Д. Об оценке решения краевых задач для неявных дифференциальных уравнений// Вестн. PAEH. - 2019. - 19, № 2. - С. 142-145.

14. Серова И. Д., Репин А. А. О существовании и оценках решений неявного дифференциального уравнения с авторегулируемым отклонением аргумента// Вестн. Тамбов. ун-та. Сер. Естеств. техн. науки. - 2018. - 23, № 123. - С. 566-574.

15. Шрагин И. В. Суперпозиционная измеримость при обобщенных условиях Каратеодори// Вестн. Тамбов. ун-та. Сер. Естеств. техн. науки. - 2014. - 19, № 2. - С. 476-478.

16. Arutyunov A. V., Zhukovskiy E. S., Zhukovskiy S. E. Coincidence points principle for mappings in partially ordered spaces// Topol. Appl. - 2015. — 179, № 1. - P. 13-33.

17. Arutyunov A. V., Zhukovskiy E. S., Zhukovskiy S. E. Coincidence points principle for set-valued mappings in partially ordered spaces// Topol. Appl. — 2016. - 201. — P. 330-343.

Жуковская Татьяна Владимировна

Тамбовский государственный технический университет

E-mail: t_zhukovskaia@mail.ru

Серова Ирина Дмитриевна

Тамбовский государственный университет имени Г. Р. Державина

E-mail: irinka_36@mail.ru 